\title{
AVALIAÇ̃̃o DA TROCA GASOSA DE PACIENTES COM INSUFICIÊNCIA CARDÍACA CONGESTIVA
}

Vanessa Emille Carvalho de Sousa ${ }^{1}$, Michelle Helcias Montoril², Lívia Maia Pascoal ${ }^{1}$, Marcos Venícios de Oliveira Lopes ${ }^{3}$

RESUMO: A fisiopatologia da Insuficiência Cardíaca Congestiva (ICC) repercute em desequilíbrios na relação ventilação-perfusão, um prejuízo que pode interferir diretamente na sobrevida dos pacientes. A equipe de enfermagem pode monitorar e prevenir esta problemática com o uso das classificações de enfermagem, no entanto, pesquisas que abordem a utilização destes instrumentos são escassas. Este estudo transversal, descritivo-exploratório, objetivou avaliar o grau de comprometimento da troca gasosa de pacientes com ICC, com base na Classificação dos Resultados de Enfermagem. Foi realizado com 37 pacientes internados em um hospital especializado, com dados obtidos por meio de entrevista, exame físico e consulta ao prontuário. Os resultados demonstram que os indicadores clínicos profundidade e frequência respiratória, ortopneia, dores no peito, sonolência e ureia foram os mais comprometidos. O desenvolvimento de pesquisas com base nos resultados de enfermagem é relevante e pode contribuir para identificação coerente dos diagnósticos de enfermagem, direcionando os cuidados ao paciente.

PALAVRAS-CHAVE: Diagnóstico de enfermagem; Troca gasosa pulmonar; Insuficiência cardíaca.

\section{EVALUATION OF GAS EXCHANGE IN PATIENTS WITH CONGESTIVE HEART FAILURE}

\begin{abstract}
The pathophysiology of congestive heart failure (CHF) reflects in imbalances in ventilation-perfusion injury that can directly affect patient survival. The nursing staff can monitor and prevent this problem with the use of nursing classifications, however, research that addresses the use of these instruments are lacking. This cross-sectional, descriptive-exploratory study aimed to evaluate the degree of gas exchange impairment in patients with CHF based on the Nursing Outcomes Classification. It was performed on 37 patients admitted to a specialized hospital with data obtained by interview, physical examination and consultation records. The results show that the clinical indicators depth and frequency of breathing, orthopnea, chest pains, drowsiness and urea were the most compromised. The development of results-based nursing researches is relevant and can contribute to consistent identification of nursing diagnoses, directing patient care. KEYWORDS: Nursing diagnosis; Pulmonary gas exchange; Heart failure.
\end{abstract}

\section{EVALUACIÓN DEL CAMBIO GASEOSO DE PACIENTES CON INSUFICIENCIA CARDÍACA CONGESTIVA}

\begin{abstract}
RESUMEN: La fisiopatología de la Insuficiencia Cardíaca Congestiva (ICC) repercute en desequilibrios acerca de la ventilación-perfusión, un perjuicio que puede interferir directamente en la "sobrevida" de los pacientes. El equipo de enfermería puede fiscalizar y prevenir esta problemática por medio del uso de las clasificaciones de enfermería, pero investigaciones que abordan la utilización de eses instrumentos son escasas. Este estudio transversal, descriptivo exploratorio tuvo el objetivo de evaluar el grado de comprometimiento del cambio gaseoso de pacientes con ICC con base en la Clasificación de los Resultados de Enfermería. Fue realizado con 37 pacientes internados en un hospital especializado con datos obtenidos por medio de entrevista, examen físico y consulta al prontuario. Los resultados demuenstran que los indicadores clínicos: profundidade y frecuencia respiratoria, ortopnesía, dolores en pecho, somnolencia y uresía fueron los más comprometidos. El desarrollo de investigaciones con base en los resultados de enfermería es relevante y puede contribuir para identificación coherente de los diagnósticos de enfermería, direccionando los cuidados al paciente.
\end{abstract} PALABRAS CLAVE: Diagnóstico de enfermería; Cambio gaseoso pulmonar; Insuficiencia cardíaca.

\footnotetext{
*Artigo desenvolvido a partir da monografia intitulada "Diagnóstico de enfermagem na troca de gases prejudicada em pacientes com insuficiência cardíaca congestiva", aprovada pela Universidade Federal do Ceará-UFC.

${ }^{1}$ Enfermeira. Mestranda pelo Programa de Pós-Graduação em Enfermagem da UFC. Bolsista CNPq.

${ }^{2}$ Enfermeira. Especialista em Enfermagem em Emergência. Enfermeira da UNIMED de Fortaleza/CE.

${ }^{3}$ Enfermeiro. Pós-Doutor em Estatística e Investigação Operativa. Professor Adjunto do Departamento de Enfermagem da UFC. Pesquisador $2 \mathrm{CNPq}$.
}

\author{
Autor correspondente: \\ Vanessa Emille Carvalho de Sousa \\ Universidade Federal do Ceará \\ R. Dezesseis, 42 - 65054-709 - São Luís-MA-Brasil \\ Email: v_emille@hotmail.com
}

Recebido: $16 / 05 / 10$

Aprovado: $15 / 11 / 10$ 


\section{INTRODUÇÃO}

A monitoração do desempenho respiratório de pacientes com Insuficiência Cardíaca Congestiva (ICC) é fundamental. O edema pulmonar e a hipoxemia, secundários ao desequilíbrio da relação ventilação-perfusão ocasionado pela doença, acarretam prejuízos tanto na oxigenação quanto na eliminação do dióxido de carbono através da membrana alvéolo-capilar ${ }^{(1-3)}$. Desta forma, a avaliação de indicadores clínicos da troca gasosa de pacientes com ICC é pertinente e pode contribuir para a identificação mais apropriada de diagnósticos de enfermagem nesta população.

Pesquisas que abordam as classificações de enfermagem são escassas no cenário brasileiro atual quanto à implementação das taxonomias de diagnósticos, intervenções e resultados de enfermagem ${ }^{(4)}$. Não foram identificados estudos nacionais que abordassem a troca gasosa de pacientes com ICC, embora a história natural e a fisiopatologia da doença indiquem este desequilíbrio ${ }^{(5-6)}$. Considera-se que a ocorrência de distúrbios na troca gasosa de pacientes cardíacos guarda relação com o tipo de lesão cardíaca e o tempo de hospitalização ${ }^{(7-10)}$. Diante destes elementos, justifica-se a realização do presente estudo.

As dificuldades para se identificar adequadamente sinais clínicos de desequilíbrios respiratórios decorrem, muitas vezes, da carência de aparelhagem e exames específicos que confirmem os achados, visto que na maioria das situações clínicas com as quais os enfermeiros se deparam, estes itens não estão disponíveis ${ }^{(10)}$. Esta situação não só dificulta a detecção de diagnósticos de enfermagem como pode mascarar uma situação clínica grave, que necessita de intervenção imediata.

Em resposta a estas lacunas, sentiu-se a necessidade de elaborar um estudo que conduza para uma sistematização da assistência de enfermagem mais ampla e ajude no direcionamento do cuidado de enfermagem. A partir daí, optou-se pela identificação de indicadores clínicos do funcionamento respiratório de pacientes com ICC, mediante a aplicação de escalas da Classificação dos Resultados de Enfermagem (NOC-Nursing Outcomes Classification).

A NOC é uma organização sistemática de resultados de enfermagem, desenvolvida em resposta à necessidade de completar a documentação clínica dos achados identificados pelo enfermeiro. Sua estrutura é abrangente e contempla diversos indicadores clínicos, isto é, variáveis específicas que reagem às intervenções de enfermagem. A estruturação em escalas do tipo Likert possibilita uma caracterização do estado de saúde do paciente em um nível mais concreto e reprodutível ${ }^{(11)}$.

Vale ressaltar que os resultados de enfermagem podem ser avaliados em diferentes momentos ao longo da aplicação da sistematização da assistência, e não apenas na etapa de Avaliação, como tradicionalmente se acredita, o que pode inclusive auxiliar na fase de identificação de Diagnósticos de enfermagem.

Como proposta de avaliação da troca de gases, a NOC apresenta sete possíveis resultados esperados e diversos indicadores do estado de saúde. Os pacientes avaliados nesse estudo enquadram-se, de forma direta ou indireta, no comprometimento cardiocirculatório, em cinco dos resultados esperados para a troca gasosa: Estado respiratório: ventilação, Perfusão tissular: pulmonar, Estado dos sinais vitais, Estado respiratório: troca de gases, e Equilíbrio eletrolítico e acido-básico ${ }^{(11)}$.

O presente estudo foi desenvolvido diante da relevância da identificação dos referidos indicadores clínicos em pessoas com ICC; objetivou-se avaliar o grau de comprometimento da troca gasosa de pacientes com ICC, com base na NOC. A aquisição de conhecimentos acerca do uso das classificações de enfermagem permite um melhor embasamento para a elaboração de um plano de cuidados específico, que subsidie ações de enfermagem mais eficazes.

\section{METODOLOGIA}

Trata-se de um estudo transversal, de caráter descritivo-exploratório, sobre o comprometimento de indicadores clínicos da troca gasosa de pacientes internados com diagnóstico médico de ICC, desenvolvido no período de agosto a outubro de 2007, em um centro de referência em doenças cardiopulmonares do Município de Fortaleza/CE.

A amostra foi composta por 37 indivíduos, selecionados por amostragem consecutiva, isto é, amostragem não probabilística, na qual os indivíduos são incluídos na medida em que se internam e atendem aos critérios de seleção do estudo. Foram definidos como critérios de inclusão: ter diagnóstico de ICC, ter sido admitido no hospital em período inferior a 48 horas e ter idade superior a 18 anos. Foram excluídos do estudo pacientes que apresentassem condições físicas ou psicológicas que impossibilitassem a coleta de dados ou que portassem patologias renais, hepáticas, 
imunológicas, ósseas, hematológicas e oncológicas que contribuem para alteração do quadro clínico específico da doença cardíaca de base.

Procederam-se a entrevista e exame físico mediante a aplicação de um instrumento contendo dados sociodemográficos, dados clínicos (variáveis referentes ao funcionamento cardiorrespiratório e variáveis antropométricas) e escalas NOC com dados relativos ao estado de saúde dos pacientes, cujos escores variam de 1 (extremamente comprometido) a 5 (não comprometido).

As escalas NOC foram escolhidas como ferramentas para a avaliação da troca gasosa dos pacientes devido ao seu potencial em reduzir a subjetividade desta avaliação, tendo em vista que o estado respiratório do paciente é classificado em níveis.

Com o objetivo de reduzir vieses, o instrumento foi submetido a um grupo de cinco especialistas na temática para validação de conteúdo e aparência do mesmo. Além disso, realizou-se um treinamento com os pesquisadores responsáveis pela coleta e um préteste para identificação de incongruências, mediante o qual o instrumento foi considerado adequado.

O projeto foi aprovado pelo Comitê de Ética em Pesquisa da referida instituição, sob Parecer n. 381/06, conforme as diretrizes da Resolução 196/96 do Conselho Nacional de Saúde, que regulamenta as pesquisas realizadas com seres humanos. Os pacientes foram esclarecidos a respeito da aplicação do instrumento, bem como do exame físico, objetivo e métodos do estudo, tornando-se conscientes do sigilo conferido às informações e identidades. A anuência foi dada mediante autorização verbal e assinatura do Termo de Consentimento Livre e Esclarecido, para cada paciente avaliado.

\section{RESULTADOS}

A análise dos dados abrangeu variáveis categóricas e numéricas, sendo consideradas as frequências absolutas e valores percentuais na avaliação das variáveis categóricas, e medidas de tendência central, desvio padrão e percentis na avaliação das numéricas. Os pressupostos para aplicação dos testes estatísticos foram avaliados pelo Teste de Kolmogorov-Smirnov, para Normalidade, e de Levene para a Homocedasticidade, sendo estabelecido o nível de significância de $5 \%$. Estes testes foram escolhidos tendo em vista que algumas variáveis da amostra apresentaram uma distribuição simétrica, enquanto outras variáveis apresentaram distribuição assimétrica.

$\mathrm{Na}$ amostra, composta por 37 pacientes, observou-se maior prevalência de indivíduos do sexo masculino $(56,8 \%)$ e que viviam com companheiro (73\%). A média de idade foi de 47,84 anos $( \pm 13,47)$, sendo alocados no percentil 75 pacientes com até 57 anos, faixa etária correspondente à fase adulta e produtiva.

Quanto aos dados clínicos obtidos a partir do exame físico dos pacientes, constatou-se que somente 8 do total de 21 variáveis avaliadas estiveram fora dos parâmetros de normalidade. Estas foram: saturação de oxigênio, porcentagem de água, massa gorda e massa magra, perímetro da cintura, relação cintura-quadril, soma das pregas, e respiração.

A tabela 1 apresenta os indicadores comprometidos moderada e substancialmente (escores 3 e 4, respectivamente). Optou-se pela avaliação dos percentis visto que o teste de Kolmogorov-Smirnov demonstra que as variáveis apresentam uma distribuição assimétrica.

Tabela 1 - Indicadores dos estados de saúde da classificação dos resultados de enfermagem em pacientes com Insuficiência Cardíaca Congestiva. Fortaleza, 2007

\begin{tabular}{lcccccc}
\hline Indicadores & Média & DP & P25 & P50 & P75 & Valor p* \\
\hline Profundidade da respiração & 3,14 & 1,09 & 2,00 & 3,00 & 4,00 & 0,236 \\
Sonolência & 3,43 & 1,34 & 2,00 & 4,00 & 5,00 & 0,038 \\
Ureia & 3,80 & 1,14 & 3,00 & 4,00 & 5,00 & 0,128 \\
Ortopneia & 3,97 & 0,73 & 3,00 & 4,00 & 4,75 & 0,035 \\
Frequência respiratória & 4,08 & 1,18 & 3,00 & 5,00 & 5,00 & 0,003 \\
Dores no peito & 4,10 & 1,51 & 2,50 & 5,00 & 5,00 & 0,002 \\
Dispneia em repouso & 4,11 & 0,51 & 4,00 & 4,00 & 4,00 & 0,000 \\
\hline
\end{tabular}

*Teste de Kolmogorov-Smirnov.

Fonte: dados primários

Conforme as pontuações identificadas na escala, observa-se que alguns indicadores dos estados 
de saúde estiveram comprometidos, o que se destaca pelo risco potencial de agravamento. A Profundidade da respiração foi o indicador mais alterado, com comprometimento substancial (escore 2,00) para $25 \%$ dos avaliados. Enfatiza-se ainda que $50 \%$ dos pacientes apresentaram um comprometimento moderado (escore 3,00$)$.

O indicador Sonolência esteve substancialmente comprometido (escore 2,00) em até $25 \%$ dos avaliados, porém, 75\% dos pacientes não demonstraram nenhum comprometimento. $\mathrm{O}$ indicador Dores no peito mostrou-se substancialmente comprometido em $25 \%$ das avaliações.

A tabela 2 apresenta as estatísticas de diferença de mediana dos indicadores clínicos avaliados, segundo sexo. Optou-se pela exposição, na tabela, somente das variáveis com diferença estatisticamente significante $(\mathrm{p}<0,05)$.

Tabela 2 - Estatísticas de diferença de mediana dos indicadores de avaliação da troca gasosa, segundo sexo, em pacientes com Insuficiência Cardíaca Congestiva. Fortaleza, 2007

\begin{tabular}{|c|c|c|c|}
\hline \multirow[b]{2}{*}{ Indicadores } & \multicolumn{2}{|c|}{ Mediana dos postos por sexo } & \multirow[t]{2}{*}{ valor $p^{*}$} \\
\hline & Masculino & Feminino & \\
\hline Profundidade da respiração & 22,90 & 12,33 & 0,002 \\
\hline Aspiração das vias aéreas & 16,07 & 22,84 & 0,014 \\
\hline Creatinina sérica & 5,25 & 10,50 & 0,007 \\
\hline Ureia & 5,64 & 10,06 & 0,042 \\
\hline
\end{tabular}

*Teste de Mann-Whitney.

Fonte: dados primários.

O teste de Mann-Whitney apontou para uma diferença de mediana $(\mathrm{p}<0,05)$ nas variáveis Profundidade da respiração, Aspiração das vias aéreas, Creatinina sérica e Ureia, de acordo com a variável sexo, em que os homens encontravam-se mais comprometidos que as mulheres, exceto para a profundidade.

A tabela 3 apresenta a estatística de correlação entre os indicadores e a idade dos pacientes avaliados, sendo expostos somente aqueles que mostraram diferença estatisticamente significativa.

Tabela 3 - Estatística de correlação entre os indicadores de avaliação da troca gasosa e a idade dos pacientes com Insuficiência Cardíaca Congestiva. Fortaleza, 2007

\begin{tabular}{lcc}
\hline Indicadores & $\mathbf{R}$ & Valor $\mathbf{p}^{*}$ \\
\hline Ritmo Respiratório & 0,27 & 0,096 \\
Orientação cognitiva & $-0,30$ & 0,073 \\
Formigamento nas extremidades & 0,48 & 0,051 \\
\hline
\end{tabular}

*Teste de correlação linear de Spearman.

Fonte: dados primários

Observa-se na tabela 3 que as estimativas das correlações lineares não foram significativas ao nível de 0,05 , ou seja, não há evidências de que haja algum tipo de relação linear entre cada indicador e a idade dos pacientes. Se utilizarmos um nível de significância de 0,1 verifica-se que a idade revelou associação estatisticamente significante com três indicadores, sendo dois lineares crescentes e um linear decrescente.

\section{DISCUSSÃO}

Segundo a Organização Mundial de Saúde, a ICC é uma das principais prioridades entre as enfermidades crônicas que necessitam de atenção dos setores de saúde em todo o mundo. A prevalência da doença aumentou nas últimas cinco décadas e considera-se que sua mortalidade pode ultrapassar $50 \%$ em cinco anos, a partir do momento do seu diagnóstico ${ }^{(12)}$.

Em nossa amostra, houve maior prevalência de indivíduos do sexo masculino. Diferenças fisiológicas entre homens e mulheres com ICC podem contribuir, em parte, para as diferenças na qualidade de vida. Mulheres portadoras de ICC são mais susceptíveis a apresentar comorbidades, como hipertensão e diabetes, e passam por um período de debilitação física, antes de morrer, maior do que em homens ${ }^{(13)}$.

Quanto à idade, verificou-se uma prevalência importante na amostra de indivíduos em fase produtiva, isto é, inferior a 60 anos, o que chama a atenção para o fenômeno da juvenilização da população acometida por doenças cardiovasculares.

A literatura mostra que, entre os pacientes 
com mais de 60 anos, a ICC é a principal causa de internação, promovendo elevados custos de internamentos e atendimentos de emergência, além de provocar perdas quanto à qualidade de vida e ao mercado de trabalho, o que resulta, muitas vezes, em altíssimos custos para o país e em aposentadorias precoces ${ }^{(14)}$. Diante disto, ressalta-se a importância das ações preventivas e da detecção precoce da doença, como estratégias de combate a esta problemática.

A avaliação clínica dos indivíduos evidenciou alterações predominantemente antropométricas, que permitiram classificar os entrevistados como portadores de sobrepeso. Estudos recentes apontam a gordura andróide como sendo um dos maiores potenciais de risco para as doenças coronarianas ${ }^{(15)}$. No presente estudo, até mesmo os pacientes com Índice de Massa Corporal e Somatório das pregas cutâneas dentro dos parâmetros normais apresentaram medidas de circunferência abdominal acima do esperado. Estes resultados refletem a problemática do aumento da prevalência de sobrepeso e obesidade na população brasileira adulta, e sua correlação com a ocorrência de cardiopatias.

A análise dos indicadores clínicos da escala NOC evidenciou o indicador Profundidade da respiração como o mais comprometido, sendo detectado um comprometimento substancial (escore 2,00) em 25\% da amostra. Enfatiza-se, ainda, que 50\% dos pacientes apresentaram um comprometimento moderado (escore 3,00 ), de maneira que estes indivíduos encontravam-se em risco potencial para prejuízos na troca gasosa.

O comprometimento do indicador Sonolência foi identificado quando alguns pacientes referiam necessidade de cochilos durante o dia, sendo mais frequentes os cochilos com duração de 1 a 3 horas, independentemente do sono noturno. Muitos pacientes referiram insatisfação do sono noturno e, por isso, aproveitavam o tempo ocioso durante o dia para repousar. Acredita-se que a mudança de ambiente, iluminação, preocupação com a saúde, hábitos de sono e posicionamento contribuem para esse resultado ${ }^{(16)}$. Desta forma, o comprometimento deste indicador em nossa amostra não pôde ser elucidado e, provavelmente, não guarda relação causal direta ou exclusiva com prejuízos no intercâmbio gasoso.

O comprometimento da frequência e da profundidade respiratória pode estar diretamente relacionado com o mecanismo patológico da ICC, que envolve dispneia progressiva aos esforços, podendo evoluir para dispneia em repouso e ortopneia. A di- ficuldade respiratória é considerada como o sintoma mais comum da doença ${ }^{(17)}$. Este mecanismo também pode ter sido o fator causal das alterações respiratórias identificadas nesse estudo.

Quanto ao indicador Dores no peito, encontram-se na literatura algumas explicações cabíveis para o sintoma, apesar de não se saber uma causa exata que esclareça a dor. Acredita-se que a redução do débito cardíaco, quando ocasiona interrupção do fluxo sanguíneo para algumas regiões do tórax, ou seja, quando há isquemia, leva à liberação de substâncias ácidas pelo músculo, o que causa dor local ${ }^{(17)}$.

Identificaram-se, ainda, algumas diferenças de gênero em relação ao perfil de comprometimento de determinados indicadores, sendo que os homens mostraram piores resultados; contudo, não foi possível elucidar esta relação.

Com relação à idade, verificou-se que, à medida que a idade aumentava, os valores na escala NOC referentes ao Ritmo respiratório e ao Formigamento nas extremidades, também aumentavam. Já em relação ao indicador Orientação cognitiva, verificou-se que, com o aumento da idade, houve um decréscimo dos valores da escala. A literatura aponta que as alterações fisiológicas da idade, na maioria das vezes, acarretam uma lentidão do ritmo respiratório ${ }^{(18)}$. Não houve comprometimento para este indicador segundo a avaliação por escores da escala NOC, provavelmente devido ao quadro estável dos pacientes avaliados.

Os resultados encontrados para o indicador Orientação cognitiva demonstram um fenômeno fisiológico relativo ao envelhecimento, de redução da orientação cognitiva ${ }^{(19)}$.

Neste ponto, existem inúmeras especulações sobre prováveis mecanismos da ICC e prejuízos na função cognitiva. O comprometimento hemodinâmico acentuado sobre o fluxo sanguíneo cerebral, não exclusivamente global, mas também regional, pode ser refletido por meio da associação entre o pior prognóstico e a clínica do paciente. Os prejuízos de memória, processamento das informações e a velocidade de respostas, encontram-se entre os inúmeros aspectos neuropsicológicos alterados em clientes com ICC, porém, não houve um completo esclarecimento da fisiopatologia das alterações cognitivas ${ }^{(20)}$.

Por fim, o indicador Formigamento nas extremidades apresentou correlação positiva com a idade, porém, isto provavelmente representa uma associação espúria, tendo em vista não existirem explicações clínicas plausíveis para tal evento. 


\section{CONSIDERAÇÕES FINAIS}

Os resultados obtidos nessa pesquisa apontam para alguns indicadores clínicos do resultado de enfermagem "Troca gasosa" que estiveram mais comprometidos na amostra de pacientes com ICC. Foram eles: Profundidade respiratória, Frequência respiratória, Ortopnéia, Dores no peito, Sonolência e Ureia.

A partir desse estudo, verifica-se que tais indicadores também podem ser considerados características definidoras sensíveis para a identificação do diagnóstico de enfermagem "Troca de gases prejudicada”, o que deixa indício para uma possível apresentação posterior.

A partir dos achados, percebe-se certa contradição nas informações encontradas nos registros de enfermagem dos pacientes com ICC avaliados, visto que o diagnóstico "Troca de gases prejudicada" foi constantemente citado por alguns profissionais na assistência a esta clientela e, no entanto, nossos resultados não evidenciaram a presença de tal diagnóstico, mas sim de uma situação de risco.

Ao correlacionarmos os indicadores do estado de saúde com o sexo, foi identificado um maior comprometimento para o homem no que se refere a Ureia, Creatinina e Aspiração das vias aéreas. Em contrapartida, para a mulher, destacou-se a Profundidade da respiração.

Ressaltam-se, também, comprometimentos relacionados à idade, a exemplo da Orientação cognitiva, que se mostrou mais alterada com o progredir da idade, um reflexo do processo natural de envelhecimento do ser humano.

As escalas NOC se mostraram como ferramentas satisfatórias que possibilitaram a avaliação dos níveis de comprometimento dos indicadores da troca gasosa dos pacientes. Esta estratégia se mostrou efetiva na elaboração de uma descrição mais objetiva do estado de saúde dos indivíduos. Reforça-se a importância do desenvolvimento de estudos longitudinais com as referidas escalas, visto que os resultados de enfermagem também devem ser medidos ao longo de um continuum, em resposta às intervenções de enfermagem.

O reduzido número de estudos desenvolvidos com pacientes com ICC tem dado pouca atenção aos resultados de enfermagem. Apesar das buscas constantes na literatura não foi possível identificar trabalhos com este tema, de forma que houve dificuldade em encontrar estudos para fazer comparações com os nossos achados.

Algumas condições que limitaram a capacidade de generalização da pesquisa foram a ampla faixa etária avaliada e a condição de estabilidade, apresentada por grande parte da amostra. Acredita-se ser de fundamental importância o desenvolvimento de estudos longitudinais sobre o tema, diante de sua relevância para um direcionamento da análise dos problemas encontrados, os quais demandam ações específicas do enfermeiro.

\section{REFERÊNCIAS}

1. North American Nursing Diagnosis Association (NANDA). Diagnósticos de enfermagem da NANDA: definições e classificações 2009-2011. Porto Alegre: Artmed; 2010.

2. Dahlen R, Roberts SL. Nursing management of congestive heart failure: part I. Intensive and Critical Care Nursing. 1995; 2(5):272-9.

3. Dahlen R, Roberts SL. Nursing management of congestive heart failure: part II. Intens Crit Care Nurs. 1995;2(6):322-8.

4. Napoleão AA, Carvalho EC. Aplicabilidade de intervenções prioritárias da NIC para o diagnóstico de enfermagem desobstrução ineficaz de vias aéreas. Cogitare Enferm. 2007;12(1):9-19.

5. Silva VM, Lopes MVO, Araujo TL. Asociación entre diagnósticos de enfermería en niños com cardiopatias congênitas. Enferm Cardiol. 2004;11(32/33):33-7.

6. Paiva G, Lopes MVO. Respuestas humanas identificadas en paciente con infarto agudo del miocardio ingresados en una unidad de terapia intensiva. Enfermería en Cardiologia. 2005;12(36):22-7.

7. Faria MFG. Diagnósticos de enfermagem respiratórios em pacientes cardíacos cirúrgicos [dissertação]. São Paulo (SP): Universidade de São Paulo; 2000.

8. Silva RCGE, Cruz DALM. Identificação dos diagnósticos de enfermagem, características definidoras e fatores de risco em pacientes valvopatas. Revista da Sociedade de Cardiologia do Estado de São Paulo. 2002;12(2):1-7.

9. Galdeano LE, Rossi LA, Nobre LF, Ignácio DS. Diagnóstico de enfermagem de pacientes no período transoperatório de cirurgia cardíaca. Rev Latino-Am 
Enfermagem. 2003; 11(2):199-206.

10. Silva VM. Caracterização de diagnósticos de enfermagem em crianças com cardiopatia congênita: estudo num hospital especializado em doenças cardiopulmonares [dissertação]. Fortaleza (CE): Universidade Federal do Ceará; 2005.

11. Johnson M, Maas M, Moorhead S. Classificação dos resultados de enfermagem (NOC). $2^{\mathrm{a}}$ ed. Porto Alegre: Artmed; 2004.

12. Tavares LR, Victer H, Linhares JM, Barros CM, Oliveira MV, Pacheco LC, et al. Epidemiologia da insuficiência cardíaca descompensada em Niterói Projeto EPICA. Arq Bras Cardiol. 2004;82(2):121-4.

13. Bennett SJ, Perkins SM, Lane KA, Deer M, Brater DC, Murray MD. Social support and healthy related quality of life in chronic heart failure patients. Qual Life Res. 2001;10:671-82.

14. Miranda CH, Castro RBP, Pazin Filho A. Abordagem da descompensação aguda da insuficiência cardíaca crônica. Medicina. 2003;36:179-86.

15. Pitanga FJG, Lessa I. Indicadores antropométricos de obesidade como instrumento de triagem para risco coronariano elevado em adultos na cidade de Salvador - Bahia. Arq Bras Cardiol. 2005;85(1):26-31.

16. Rocha LA, Maia TF, Silva LF. Diagnósticos de enfermagem em pacientes submetidos à cirurgia cardíaca. Rev Bras Enferm. 2006;59(3):321-6.

17. Guyton AC, Hall JE. Tratado de fisiologia médica. $9^{\mathrm{a}}$ ed. Rio de Janeiro: Guanabara; 1997.

18. Netto FLM. Aspectos biológicos e fisiológicos do envelhecimento humano e suas implicações na saúde do idoso. Pensar Prát. 2004;7:75-84.

19. Santos WL, Nakatani AYK, Santana RF, Bachion MM. Diagnósticos de enfermagem identificados na alta hospitalar de idosos. Cogitare Enferm. 2009; 14(2):304-10.

20. Bennett SJ, Sauve MJ. Cognitive deficits in patients with heart failure: a review of the literature. $J$ Cardiovasc Nurs. 2003; 18:219-42. 